\title{
Ein neues Fragment von ,Unser vrouwen klage‘
}

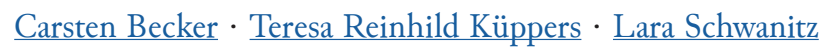

I m Herbst 2017 wurde in der Herzog August Bibliothek Wolfenbüttel unter der Signatur Cod. Guelf. 346 Noviss. $8^{\circ}$ ein Fragment inventarisiert ${ }^{\mathrm{I}}$, das aus dem Nachlass Gustav Milchsacks stammt. Eine erste Beschreibung des Doppelblatts veröffentlichte Christian Heitzmann in der Handschriftendatenbank der Bibliothek. ${ }^{2}$ Das Fragment, ein Doppelblatt aus einer Pergamenthandschrift im Oktavformat, umfasst 92 Reimpaarverse, die in Christian Heitzmanns Beschreibung als die Verse 502-593 aus ,Unser vrouwen klage' (Redaktion II)³ identifiziert sind. Da das Doppelblatt den Text lückenlos fortlaufend überliefert, handelt es sich um eine Lagenmitte. Die vier mit einer Größe von I25 $\times 95 \mathrm{~mm}$ auffallend kleinen Seiten sind blindliniert ${ }^{4}$ und einspaltig auf einem Raum von etwa $95 \times 60$ $\mathrm{mm}$ beschrieben. Die Verse sind abgesetzt und zusätzlich durch Reimpunkte getrennt, was eine ältere Vorlage ohne abgesetzte Verse vermuten lässt. Die Versalien sind alternierend gestaltet, wobei die Anverse durch rote und deutlich vergrößerte Anfangsbuchstaben hervorstechen, während die Anfangsbuchstaben der Abverse schwarz mit Rubrizierung ausgeführt sind. Die Ränder des Doppelblatts sind orthogonal zum Text von mindestens vier jüngeren Händen beschrieben. Heitzmann transkribiert Domine veni ad nos quia sumus mortales (B1. 2r), Universi populi omnes iam gaudete in honorem marie virginis (Bl. 2v) sowie unzusammenhängende Schriftproben (Sanctus, Maria $\bar{m} r$ gracie [...]) in verschiedenen Größen (B1. 2r)'s. Am oberen und äußeren Rand finden sich Punktierungen und eine moderne Bleistiftfoliierung.

Milchsack war das zur Diskussion stehende Fragment seit spätestens I89o bekannt, wie sich aus einer von ihm angefertigten und datierten Transkription schließen lässt. In seine 1878 veröffentlichte Textausgabe ${ }^{6}$ von ,Unser vrouwen klage‘ ging es hingegen noch nicht ein. Auch die 2017 durch Edgar Büttner abgeschlossene Neuausgabe ${ }^{7}$ konnte das wenig später wiederaufgefundene Fragment nicht mehr berücksichtigen.

Die Schrift des Fragments ist als Textualis auf mittlerem Schriftniveau zu klassifizieren. Da die Schriftentwicklungen der zweiten Hälfte des I3. Jahrhunderts deutlich erkennbar bereits vollzogen sind, ist eine Entstehung vor dem Beginn des I4. Jahrhunderts auszuschließen. Die Schrift weist charakteristische Formen auf, die verstärkt ab dem zweiten Viertel des I4. Jahrhunderts auftreten.

Das $a$ ist durchgehend doppelstöckig und geschlossen ausgeführt. Dabei ist das a stark überhöht, teilweise bis auf gleiche Höhe mit den Oberschäften von $t$, was auf das fortschreitende zweite Viertel des I4. Jahrhunderts oder sogar einen Zeitraum ab der Jahrhundertmitte hinweist. ${ }^{8}$ Der Unterbogen des $g$ ist einfach gebrochen, bis zur Mittellinie hochgezogen und bereits leicht verkümmert. Das $g$ ist noch nicht völlig in das Mittelband integriert, wie es gegen die Jahrhundertmitte insbesondere auf erhöhtem Schriftniveau zu erwarten wäre. ${ }^{9}$

Das runde Schluss-s ist aus winkligen Federstrichen zusammengesetzt. Es ist teilweise bereits zur Brezelform geschlossen. Entgegen der Tendenz zur überhöhten Schreibung des runden Schluss-s im zweiten Viertel des I4. Jahrhunderts ${ }^{\mathrm{I}}$ ragt der Buchstabe im vorliegenden Fragment oft ein kleines Stück unter die Mittellinie.

Der Bogen des $b$ ist unter die Zeile verlängert. Die Verlängerung des Bogens ist als schwungvoller Bogen nach links unter den Buchstabenkörper gestaltet und zeigt in vielen Fällen eine konkave Einwölbung. Diese Buchstabenform mit konkaver Einwölbung lässt sich insbesondere im bairisch-österreichischen Raum ab den zoer Jahren des I4. Jahrhunderts vermehrt nachweisen. ${ }^{\text {II }}$ 
Auffallend sind die rechtwinklig angeschlossenen, auf der Zeile sitzenden Schaft- und Fußstriche bei $b, o$ und $l$, die in der Textura gebräuchlich sind, aber erst ab der Jahrhundertmitte auch in der einfacheren Textualis auftreten (vgl. bspw. geboren, 512). ${ }^{12}$ Ein weiteres Merkmal für die zweite Jahrhunderthälfte ist die relative Gleichgestaltung von $m, n$ und $i$, die durch gleichförmige Umbrechungen der Schäfte erreicht wird. ${ }^{13}$

Insgesamt kann das Fragment aufgrund des paläographischen Befundes auf die Mitte, vielleicht eher das zweite Drittel des I4. Jahrhunderts datiert werden.

Die Schreibsprache des Fragments weist Merkmale auf, die eine Einordnung im Südwesten des mittelbairischen Sprachgebiets nahelegen. Das heutige Nordtirol erscheint plausibel. Generell ostoberdeutsche Merkmale sind die Diphthongierung der mhd. Langvokale $\hat{u}$ und $\hat{\imath}$, letztere auch im Derivationssuffix $-l \hat{\imath} c h,{ }^{14}$ sowie die konsequente Verwendung von $c b$ für mhd. $k$ in allen Positionen, ${ }^{15}$ konsequente Schreibung der Auslautverhärtung von mhd. $-c$ und $-p^{16}$ und Vorherrschen der bairischen Kennform schol. ${ }^{17}$ Markant ist ferner die Verwendung von $z z$ für mhd. $z$ nach Langvokal und Diphthong. ${ }^{18}$ Darüber hinaus gibt es Hinweise für die Dehnung von mhd. $i$ vor $r$, die Paul vorsichtig als gesamtbairisches Phänomen auffasst. ${ }^{19}$

Ausgewählte Belege: zu mhd. $\hat{\imath}$ : dein/dein ( $502,527,5 \varsigma 1) ;$ leipl leibes (579, 582); mein/meine (505, 525, 552); puterleichen (584); fcheffelein (509). Zu mhd. $\hat{u}$ : auz (568); zefaure (538); dagegen nu (532, 56I). - Zu mhd. iu: baidiu (566, 583); div (56I, 575); liebiu $(525,558)$; triwen $(552) . \quad-\mathrm{Zu}$ mhd. $k$ : bechomen (545); cher (54); chomen/chumt (518, 542); manich (58I); trinchen/ tranch (517, ,18). - Zu mhd. soln: schol//cholt (506, 531, 549); dagegen fol (522, 555). - Zu mhd. 3: geniezzen (555); grozzem (586); Suezziu (528). - Zur Dehnung von i: dier ( $515,534,553)$; mém (516). ${ }^{20}$

Eher in den Westen des Bairischen weisen einige Ausnahmen zu den oben genannten Merkmalen. Bezüglich der Diphthongierung ist auffällig, dass mhd. iu im Fragmenttext nur als iu iv iw erscheint, obwohl die Diphthongierung von mhd. iu sehr früh eingetreten sein soll; ${ }^{21}$ umso interessanter ist, dass sie sich im Text zumindest in der Graphie nicht zeigt. In Urkunden des I3. Jahrhunderts kommen $\mathrm{eu}$-Schreibungen im Nebenton westlich der Isar allerdings ebenfalls kaum vor. ${ }^{22}$

Ein weiteres Merkmal, das nach Urkundenbefund tendenziell nach Bayern weist, betrifft

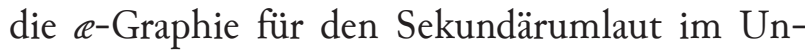

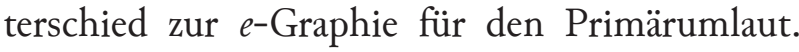
Dieses Merkmal gilt als typisch bairisch, strahlt allerdings auch in den angrenzenden schwäbischalemannischen Raum aus. ${ }^{23}$ Im Vokabular ist neben dem Schwanken zwischen sol und schol interessant, dass der Text welt ( $\mathrm{I}_{1}$ ) verwendet statt ostoberdeutsch werlt. ${ }^{24}$ Auffällig ist auch die Form geein (510).

Paul charakterisiert die Form gesin für mhd. gewesen als alemannisch, ${ }^{25}$ doch erscheint sie hier mit eher östlicher Diphthonggraphie, wobei allerdings Reimzwang mit fchaffelein (509) nicht auszuschließen ist.

Belege: zum Primärumlaut: ende (565); engel (548), engellich/chen (504); bende (566); menfch ( $5 \mathrm{II})$; fellselel Jelen (508, 519, 522); fwere (575); zeher (569). - Zu mhd. a: Chaffelein ( 509$)$.

Eine weitere Ausnahme, die zumindest aus dem bairischen Kerngebiet hinausweist, hängt mit der Auslautverhärtung zusammen. Deren graphische Repräsentation fehlt sekundär in Kontexten, in denen die Apokope eingetreten ist. Diese ist im vorliegenden Fragment jedoch nicht vollständig durchgeführt: Das Verhältnis der apokopierten zu nicht apokopierten Formen beträgt etwa 2 zu 3. Dem paläographischen Befund nach weist das vorliegende Fragment Merkmale des zweiten Drittels des I4. Jahrhunderts auf. Laut Lindgren zeigt sich die Apokope zu dieser Zeit im Bairischen nahezu durchgängig, doch erreicht die Apokope in der Graphie beispielsweise im Schwäbischen erst um I375 die 5o-Prozent-Marke, sodass eine Verortung weiter nach Westen auch vor diesem Hintergrund nicht auszuschließen ist. ${ }^{26}$ 
Der Diphthongwandel des mhd. Diphthongs $e i$ ist konsequent durchgeführt: Mhd. ei erscheint fast durchgängig als ai mit Ausnahme von zwei Stellen, an denen nur $a$ steht, so in an (565) und blach (577) - Büttner ediert an diesen Stellen ein bzw. blaich. ${ }^{27}$ Die ai-Graphie ist ein typisch bairisches und ostschwäbisches Merkmal. ${ }^{28}$ Wiesinger gibt zudem Belege für die ab dem frühen I2. Jahrhundert nachweisbare inselhafte Entwicklung von mhd. ei zu $a$ in Teilen des Mittel- und Südbairischen an. ${ }^{29}$ Darüber hinaus lässt sich noch ein Vorkommen von zebafe für mhd. ze belfe, zu Hilfe finden, bei dem $l$-Vokalisierung vorliegen könnte. Nach Paul ist die Vokalisierung von $l \mathrm{zu} i$ im Auslaut ein mittelbairisches Phänomen, das „um 1300"30 auftrat; mhd. el fällt hier also mit mhd. ei zusammen. Mhd. el würde demnach sekundär auch vom Zusammenfall mit mhd. a betroffen, was die Graphie hafe für mhd. belfe erklären könnte. Aufgrund der bisherigen Beleglage kommt von den bei Wiesinger genannten Inseln Tirol im I3./I4. Jahrhundert dafür in Frage. ${ }^{31}$

$\mathrm{Zu}$ diesem Befund passt auch die auffällige Repräsentation sowohl von mhd. kurzem $o$ als auch lan- gem $\hat{o}$ durch die Graphien oe und $\stackrel{e}{o}$. Hieran schließt sich der Umlaut von mhd. $\hat{o}$ an, der ebenfalls in beiden auftretenden Fällen als $\stackrel{\varrho}{o}$ erscheint. Nach Paul ist der Zusammenfall von mhd. ô und $\propto$ im I3. Jahrhundert ein typisches Merkmal für das Mittel- und Südbairische. ${ }^{32}$

Belege: zu mhd. o: erchorrn (536); geboern (535), verloern (5II); dagegen geboren ( $(13)$. - Zu mhd. o: floez (568, 569); groez $(567)$, groz (553); noet (514, 584), net (576); toet $(538,583)$, toot (513, 516, 522, 529, 575); trone (505); dagegen schone (Adv.) (506).

Insgesamt weist der Fragmenttext also ein deutlich bairisches Gepräge auf. Ein Zusammenfall von ei mit a sowie $l$-Vokalisierung deuten sich als mittel- bzw. südbairische Merkmale zumindest an, markant ist die ebenfalls mittel- bzw. südbairische $\stackrel{\varrho}{o}$-Graphie für mhd. $o$, $\hat{o}$. Aufgrund dieses Merkmalprofils ist eine Herkunft aus dem Südwest-Mittelbairischen (NordTirol) zu erwägen. Nach der Ausgabe von Büttner zu schließen, weist der Fragmenttext im Wortbestand zudem große Nähe zur Innsbrucker Handschrift $\mathrm{O}$ auf. ${ }^{33}$

Der Text des Fragmentes wird handschriftengetreu wiedergegeben. Lombarden werden durch Fettdruck gekennzeichnet. Am rechten Rand wird die Zählung der Ausgabe Büttner [Anm. 3] mitgeführt.

B1. Ir, I La vrawe můter dein clag.

502

Dar nach fol ich zehimel varen.

Sit den engellichfchen fcharen.

Zìe meinef vater tròne.

Da fchol ich leben fchone.

O můter la dein wainen seìn .

Wan ich die fele funden han.

Vnd auch mein liebes fchæffelein.

Daz lange irre fe ift gefein.

Der menfch lange was verloern.

Dar zv wart von dir geboren.

daz ich allain difen tôt.

Lit auch fur der welt noet.

war vm miffe ualt dier.

Sein vater toet ia hat der vater meirn.

Geboten daz ich trinchen fchol.

Der marter tranch daz chumt wol. 
Den felen die gebunden

Sint mit den helle hunden.

Den wil ich zehilfe chomen

Mein tót fol maniger fel frumen .

Da von vil zartiu můter mein

Maria la dein wainen fein.

B1. Iv, I Ach herzen liebiu fchóniv maget

Hab ain herze unverzaget

La dein wainen vber mich.

Suezziu muoter ftrófte dich .

5

Swie daz der toet an mir gefige.

Vnd fwie ich im vnder gelige.

Des fcholt tu dich niht miffe haben.

Wan fwie ich nu werde begraben.

Noch wil ich niht uer geffen dein.

IO

Wan ich wil immer mit dier fein.

Dv waift wol wie ich bin geboern.

Du bift div erwelt vnd er chơrn.

Vor aller creature.

Mein toet ift dir zefavre.

$\mathrm{Nv}$ worden vn̄ wirt noch me.

Dir ift nach mir we vñ we.

Ez ift zeit daz cher wider.

Von dem ich pin chomen her nider.

Daz ift mein vater here.

20 Zuo dem ich wider chere.

Dar mach tu fo fchier niht bechomen.

Swie daz ich werd dir benomen.

Ain weil doch fcholt tu dar.

B1. 2r, I Chomen mit der engel fchar.

Da fcholt tu immer mit mir fein.

Maria la deinf herzen pein.

Die weil fchol iohannes dein.

Mit triwen phlegen muoter mein

Er fchol dier dienen allen weis.

Reht als dv fein můeter feis.

Mich fol er des geniezzen lan.

Daz ich in ie gemint han.

$\mathrm{V} \bar{n}$ noch von herzen minne.

Johannes liebiv minne. 
Johannes iunger gůeter .

Sich an dein můeter.

560

$\mathrm{Nv}$ nim ir war div mich geber.

I5

Si was mein mueter vndzen her.

$\mathrm{Nv}$ fchol fi dein müter fein.

$\mathrm{Nu}$ fplig ir wol reht als mein.

Do was der wort an ende.

Si wnden baidiu ir hende.

20

Jr vngehab was also groez.

Daz in auz ir augen floez.

Der zeher floez alfam ein pach.

Si fwigen ir ietwederf ßprach.

B1. 2v, I Nie ain wort fi mohten niht.

Gereden von der angefiht.

Die fi an im fahen.

Do im begunde nahen.

5

Div fwere vnd der biter tót .

Da von wart gró ir herzen noet.

Si wrden blach gel vñ val.

Jr herzen lieb hiench vor in fal.

Si fahen feinen leip gar blaich

IO

Nv waz gefwigen ir freuden laich.

Div clag div was fo manich valt.

Daz von des leibes vngewalt.

Si waren baidiv alfo toet.

Von der puterleichen noet

I5

Die $f 1$ an ir herzen.

Liten von grozzem fmerzen.

Sam ein fwert durch fi fteche.

Da von ir herze preche.

Si waren baidiv an craft.

20

Div clag het fi gar behaft.

Daz ir ietwerdes moht wort.

Gelaiften ftimme noch wort

Doch fwer es reht merchen wil

\section{Apparat}

504 Sit] Wohl Schreibfehler für Mit] vgl. Büttner [Anm. 3]. 507 sein ] Übergeschriebener Buchstabe vermutlich Verbesserung des Reims von sein zu san? 5IO $\left.\int e\right]$ Durchgestrichen und unterpungiert. $5 \mathbf{1 6}$ mein $]$ Übergeschriebene Buchstaben vermutlich Verbesserung des Reims von mein zu mier? 


\section{Kontakt}

Carsten Becker · Teresa Reinhild Küppers

Philipps-Universität Marburg · Institut für Deutsche Philologie des Mittelalters · Deutschhausstr. $15 \cdot 35037$ Marburg E-Mail: carsten.becker@uni-marburg.de·teresa.kueppers@uni-marburg.de

Lara Schwanitz

Universität Köln · Institut für Deutsche Sprache und Literatur I · Zülpicher Str. 47b · 50674 Köln

E-Mail: 1.schwanitz@uni-koeln.de

\section{Anmerkungen}

I Digitalisat online: <http://diglib.hab.de/mss/346-noviss-8f/start.htm>.

2 Christian Heitzmann, Beschreibung von Cod. Guelf. 346 Noviss. $8^{\circ}$, online: $<$ http://diglib.hab.de/?db=mss\&list=ms \&id=346-noviss-8f\&catalog=Heitzmann>.

3 Die Zählweise folgt der Ausgabe Edgar Büttner (Hg.), Unser vrouwen klage / Der Spiegel (Altdeutsche Textbibliothek I24), Berlin/Boston 2017. Büttner greift bei seiner Neuedition auf die Vorarbeiten zurück, die im Rahmen seiner 1987 veröffentlichten Dissertation entstanden sind (Edgar Büttner (Hg.), Die Überlieferung von „Unser vrouwen klage“ und des „Spiegel“, Erlangen 1987).

4 Vgl. Heitzmann [Anm. 2].

5 Vgl. Heitzmann [Anm. 2].

6 Gustav Milchsack, Unser vrouwen klage, in: Beiträge zur Geschichte der deutschen Sprache und Literatur 5 (I878), S. $193-357$.

7 Vgl. Büttner [Anm. 3].

8 Karin Schneider, Gotische Schriften in deutscher Sprache, II. Die oderdeutschen Schriften von I300 bis I350, Wiesbaden 2009, S. 88, 93, I00.

9 Vgl. Schneider [Anm. 8], S. 93.

Io Vgl. Schneider [Anm. 8], S. 89, I00.

II Vgl. Schneider [Anm. 8], S. 88, Ior.

I2 Vgl. Schneider [Anm. 8], S. 87.

I3 Karin Schneider, Paläographie und Handschriftenkunde für Germanisten. Eine Einführung (Sammlung kurzer Grammatiken germanischer Dialekte B. Ergänzungsreihe Nr. 8), Tübingen I999, S. 5I.

I4 Vgl. Hermann Paul, Mittelhochdeutsche Grammatik, 25. Auflage, neu bearbeitet von Thomas Klein, Hans-Joachim Solms und Klaus-Peter Wegera. Mit einer Syntax von Ingeborg Schröbler, neubearbeitet und erweitert von Heinz-Peter Prell (Sammlung kurzer Grammatiken germanischer Dialekte A. Hauptreihe Nr. 2), Tübingen 2007 , $\S \mathrm{L} 23$.

I5 Vgl. Paul [Anm. I4], §§ L I03, L I05.

I6 Vgl. Paul [Anm. I4], § L 72.

I7 Vgl. Karin Schneider, Gotische Schriften in deutscher Sprache, I. Vom späten I2. Jahrhundert bis um I300, Textband, Wiesbaden 1987 , S. I80.

I8 Vgl. Paul [Anm. I4], § L 6i.

19 Vgl. Paul [Anm. I4], § L 32.

20 Hier liegt eine Korrektur vor, die, entsprechend dem dier in der darüberliegenden Zeile, als mier aufzulösen ist.

2i Vgl. Paul [Anm. I4], § L I7. 
22 Vgl. Corpus der altdeutschen Originalurkunden bis zum Jahr 1300, hg. von Friedrich Wilhelm u. a., 5 Bände. Lahr/Berlin 1932-2004.

23 Vgl. Paul [Anm. I4], § L 38.

24 Den mhd. Urkunden des I3. Jahrhunderts zufolge schwankt das Bairische zwischen sol und schol. Karl Weinhold, Mittelhochdeutsche Grammatik, Paderborn I883, § 213, gibt darüber hinaus zur Auskunft, dass welt im Bairischen des I4. Jahrhunderts „schon häufig“ sei. Diese Form ist hier also nicht zwingend als Alemannismus anzusehen.

25 Vgl. Paul [Anm. I4], § M I07.

26 Vgl. Kaj B. Lindgren, Die Apokope des mhd. - $e$ in seinen verschiedenen Funktionen, hg. von Pekka Katara (Suomalaisen Tiedeakatemian toimituksia - Annales academiae scientiarum fennicae Ser. B Tom. 78,2), Helsinki 1953, S. 178 .

27 Vgl. Büttner [Anm. 3], S. 91, 93.

28 Vgl. Paul [Anm. 14], § L 45.

29 Vgl. Peter Wiesinger, Zum Problem der Herkunft des Monophthongs a für mittelhochdeutsch FJh., in: Sprachgeschichte, Dialektologie, Onomastik, Volkskunde. Beiträge zum Kolloquium am 3./4. Dezember 1999 an der Johannes Gutenberg-Universität Mainz. Wolfgang Kleiber zum 70. Geburtstag, hg. von Rudolf Bentzinger u.a. (Zeitschrift für Dialektologie und Linguistik. Beiheft II5), Stuttgart 200I, S. 9I-I26.

30 Vgl. Paul [Anm. I4], § E 26,5.

3I Vgl. Wiesinger [Anm. 29], S. Ing.

32 Vgl. Paul [Anm. I4], §§ E 27,7 und L 4I-42 sowie Ingo Reiffenstein, Aspekte einer Sprachgeschichte des Bayerisch-Österreichischen bis zum Beginn der frühen Neuzeit, in: Sprachgeschichte. Ein Handbuch zur Geschichte der deutschen Sprache und ihrer Erforschung, hg. von Werner Besch, Anne Betten, Oskar Reichmann und Stefan Sonderegger, 3. Teilband, 2. vollständig neu bearbeitete und erweiterte Auflage (Handbücher zur Sprach- und Kommunikationswissenschaft 2), Berlin/New York 2003, S. 2889-2942, hier S. 2913.

33 Michael Curschmann (Hg.), Der Münchner Oswald. Mit einem Anhang: Die ostschwäbische Prosabearbeitung des I5. Jahrhunderts (Altdeutsche Textbibliothek 76), Tübingen 1974, S. XXIII-XXIV, attestiert dem ,Klage'-Abschnitt der Handschrift O eine bairisch geprägte Schreibsprache nach schwäbisch-alemannischer Vorlage. 